\title{
A MULTI-LASER SYSTEM FOR A FAST SAMPLING THOMSON SCATTERING DIAGNOSTIC
}

\author{
by \\ P.K. Trost, T.N. Carlstrom, \\ J.C. DeBoo, C.M. Greenfield, \\ C.L. Hsieh, and R.T. Snider
}

This is a preprint of a paper to be presented at the 8th Topical Conference on High Temperature Plasma Diagnostics, May 7-10, 1990, Hyannis, Massachusetts, and to be printed in the Proceedings.

\author{
Work supported by \\ Department of Energy \\ Contract DE-AC03-89ER51114
}

GENERAL ATOMICS PROJECT 3466
OCTOBER 1990

* general atomics 


\section{A multi-laser system for a fast sampling Thomson scattering diagnostic}

P.K. Trost, T.N. Carlstrom, J.C. DeBoo, C.M. Greenfield, C.L. HSIEH, AND R.T. SNIDER

General Atomics, San Diego, Califormia 92186-9084

A multi-laser system is being developed for the DIII-D Thomson scattering diagnostic. This system combines the beams from up to eight Nd:YAG lasers onto a common beamline in which the beams are nearly parallel and are all focused into a small, common area within the desired scattering volume. Each laser can be fired at a constant rate $(20 \mathrm{~Hz}$ per laser) for a high average repetition rate, or togetleer in a "burst", which will give very high sampling rates $(10-20 \mathrm{kHz})$ for short periods. The burst mode will be triggerable by plasma events, which will allow for study of transient phenomena, but will require non-periodic firing of the lasers. Beamline diagnostics include position sensitive detectors for computer controlled feedback alignment of the $35 \mathrm{~m}$ beamline, an image position detection system for monitoring the alignment of the collection lens to the scattering volume, and a 1-D reticon camera for divergence monitoring. The effects of the non-periodic firing of the lasers will be monitored with the reticon camera. 


\section{INTRODUCTION}

Thomson scattering is regarded as a primary diagnostic for the measurement of the electron temperature and density on most large fusion experiments. Since the crosssection for Thomson scattering is relatively small, very high power lasers are needed to obtain sufficient signal. High power ruby lasers were the first light source which made Thomson scattering measurements possible. ${ }^{1}$ For most large fusion experiments, the discharge period is on the order of 1-20 s. Even at periods of a second, the plasmas can vary dramatically. Single shot Thomson scattering measurements are inadequate for these experiments since many individual plasma discharges are needed to obtain a time dependent scan. Ruby lasers are still the standard light source for most Thomson scattering systems. However, by the nature of the ruby crystal, only very slow repetition rates at high power are possible. When appropriate detectors became commercially available, Nd:YAG lasers became a viable alternative to the Ruby laser for Thomson scattering measurements. ${ }^{2}$ Nd:YAG lasers have a definite advantage over Ruby lasers in that they have repetition rates of up to $100 \mathrm{~Hz}$ at high powers. However, many of the events that occur in a tokamak experiment can be on the time scale of milliseconds or less. To investigate transient phenomena, a laser system which is faster than what is presently commercially available in a single laser unit is needed.

The approach presented here uses multiple lasers and combines their beams onto a common path. The lasers may be fired with constant spacing for a high average sampling rate, or in a "burst" which will give a very high sampling rate, but at the expense of a lower steady state sampling rate. In principle, the sampling 
rate in burst mode could be arbitrarily high, but practical considerations, such as digitizer readout speed, restrict this rate. The laser system for the DIII-D multipulse Thomson scattering apparatus is being designed for eight, $20 \mathrm{~Hz}$ lasers, giving the highest steady-state sampling rate of $160 \mathrm{~Hz}$. For the burst mode, the fastest sampling rate is $10-20 \mathrm{kHz}$, limited by the digitizer readout rate, with a complete burst possible every $50 \mathrm{~ms}(20 \mathrm{~Hz})$.

\section{LASER SYSTEM}

The beam packing scheme is shown schematically in Figs. 1 and 2. On the laser table, (Fig. 1) a matrix of beams 2 wide by 4 high is constructed. The mirrors which pack these beams have full clear aperature on the edges which are adjacent to other beams. The packing is done such that the lower sections are filled at the front of the table (beam exit end) with the higher sections being filled from behind (Fig. 2). Therefore, the packing mirrors only have beams passing overhead, which allows for mounting the mirrors to simple vertical posts. The beams from either side cross over the center of the table and are packed into the opposite sides. Therefore, the portion of the mirror which is adjacent to the other beams has the substrate situated such that both the front surface and edge slope away from the adjacent beam at a 45 degree angle. This layout provides an arrangement in which the thickness of the mirror substrate and associated support structure will not interfere with other beams.

The beam pack passes from the table and traverses approximately $25 \mathrm{~m}$ to a lens which focuses it to the scattering volume within the vacuum vessel. One approach to focus the pack is to a single spot within the vessel. This would require that the 


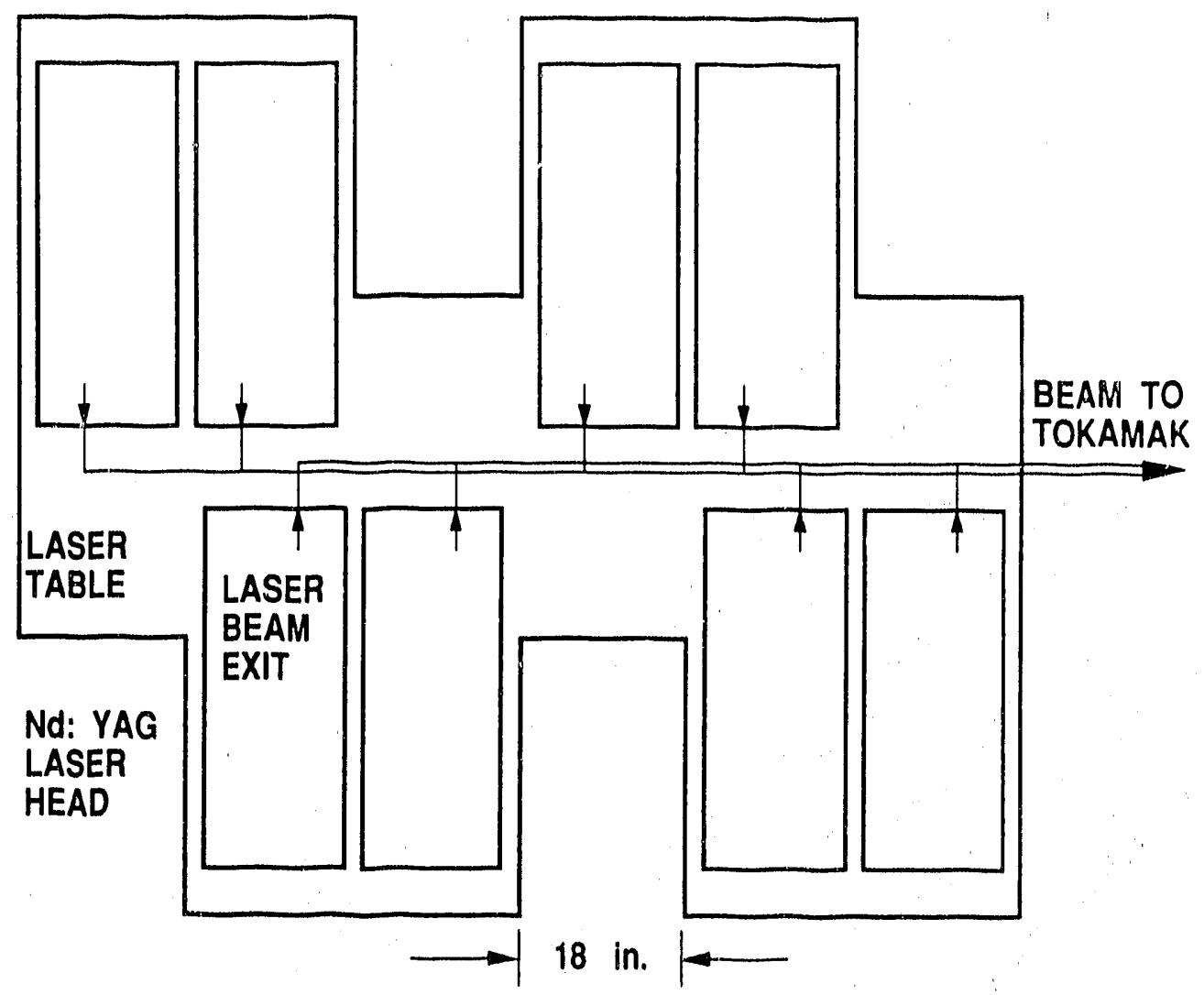

Fig. 1. Plan view of laser table with Nd:YAG lasers and beamlines.

beams be exactly parallel, and the spot would be at a position determined by the lens position and focal length. The beams would overlap only at the focal point, and would approach the focal point from different angles, so that each beam has a different angular relation to the collection lens, making alignment difficult, if not impcssible. This arrangement results in a fairly large cross-section for the beam pack nearby the lens, which would result in access problems for the beam pack unless significant modification to the existing hardware on the DIII-D vessel is done.

By overlapping selected beams at this lens, the focus points of the individual beams can be positioned behind each other (Fig. 3), and alignment problems are reduced. For the eight laser system presented here, the four up-down beams will 

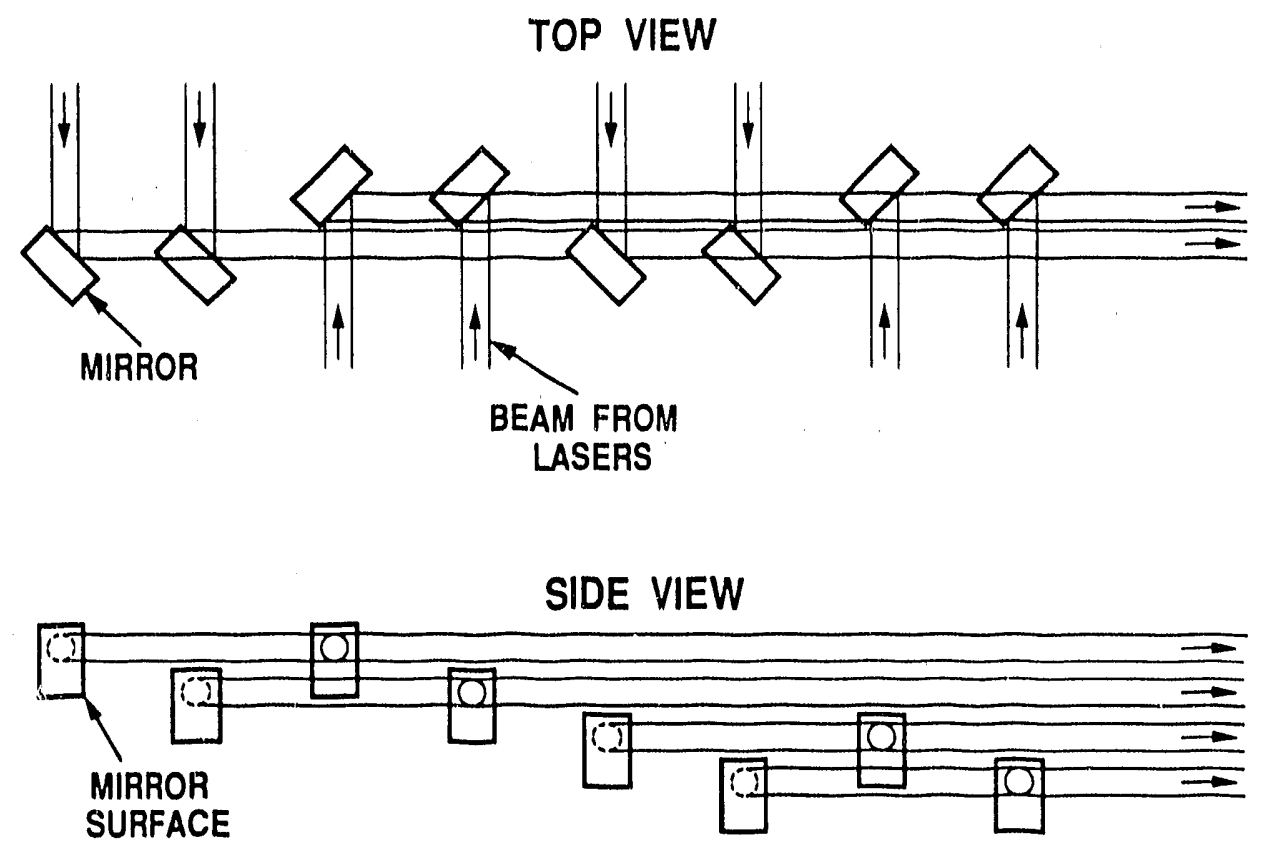

Fig. 2. View of beam packing at laser table. Beams cross over centerline and form the columns of the beam pack. The rows are formed by filling from top (end of table) to bottom (beam exit end).

overlap at the focusing lens, and each pack of four beams will be positioned so they are parallel. This will provide four sets of overlapping beams, as seen by the collection lens. The separation between the beams is very small $(6 \mathrm{~mm}$ total) compared the distance to the collection lens $(400 \mathrm{~mm})$, so the defocusing of the beam image should be minimal. The angular displacement of the beams will be symmetrical about a centerline, which will minimize alignment difficuities. This arrangement also minimizes the cross-sectional area of the beam pack which makes beam access into the vacuum vessel easier.

After the laser beam has transversed the plasma, it must be dumped (absorbed) with a minimum amount of backscatter. The laser beam dump for this system must also be able to withstand an average 160 watt load, delivered in $100 \mathrm{MW}$ pulses, 


\section{SIDE VIEW}

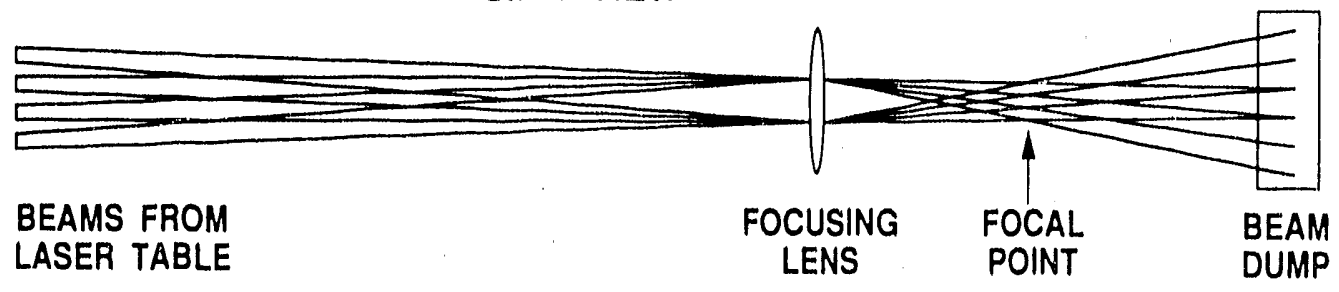

FRONT VIEW

(AS SEEN BY COLLECTION LENS)

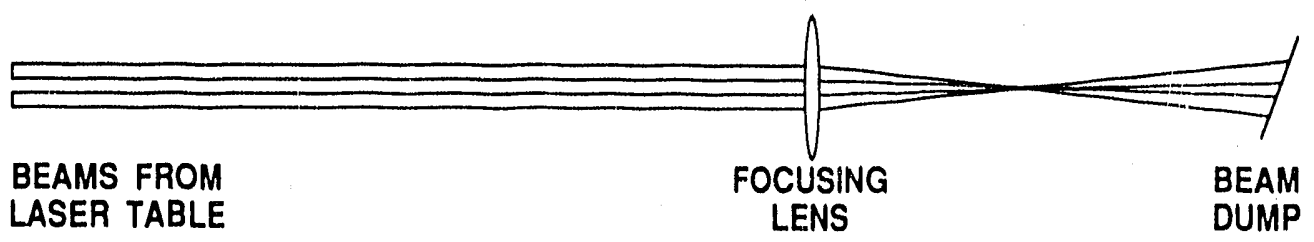

Fig. 3. Ray trace of laser beams from laser table output to the beam dump. From the perspective of the collection lens, there are only two overlapping beams (front view). Beam displacement along the collection lens' optical axis is kept within the lens' focal depth.

each on an area of approximately $2 \mathrm{~cm}^{2}$. The design used consists of a $1 \mathrm{~mm}$ thick, tempered, neutral density glass filter, mounted to a water cooled aluminum heat sink with thermally conductive paste. The neutral density filter is dyed in the melt, so that the laser beam energy is evenly deposited in the glass. Tempering the glass gives it additional resistance to fractures due to the thermal stresses caused by the heat from the laser beams. The filter glass is mounted at a 45 degree angle to the laser beam, with another neutral density glass filter mounted at an angle to the beam reflected from the first filter (Fig. 4). The optical density of the filter is chosen so that $95 \%$ of the beam is absorbed on the first pass though the filter glass. Since glass has a low coefficient of thermal conductivity, it must be kept thin to keep the heat conductivity high and thereby prevent melting of the glass. 


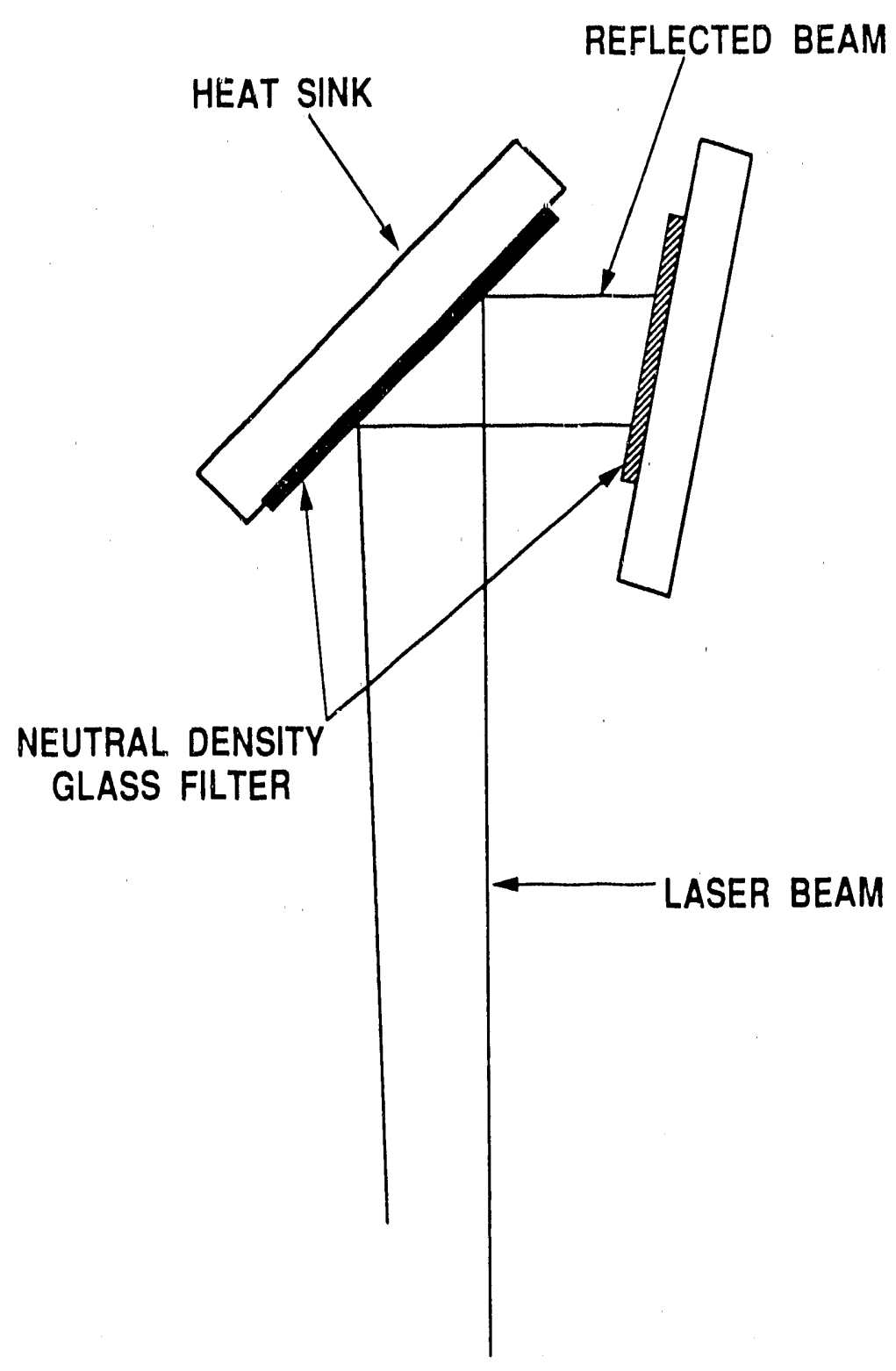

Fig. 4. Water-cooled, 160 watt, Nd:YAG laser beam dump. Thin glass filters absorb the laser power and conduct the heat rapidly into aluminum heat sink.

To further the experimental versatility of this system, a scheme to implement asynchronous trigging of the burst mode firing of the lasers is being developed. Since the burst mode is very limited in its temporal extent, a physics based trigger is 
needed to realize the fullest utilization of this feature. To achieve this, each laser power supply is built so that it can recharge in less than $20 \mathrm{~ms}$. To maintain proper thermal lensing of the $\mathrm{Nd}$ Y YAG rod, an average repetition rate of $20 \mathrm{~Hz}$. must be maintained. Therefore, the occasional burst can only be a small discontinuity from the nominal $20 \mathrm{~Hz}$. If the lasers are being fired at even intervals of $6.3 \mathrm{~ms}(160 \mathrm{~Hz})$, generally 5 of the 8 lasers in this system will be ready to fire in the burst. After the burst, periodic firing of the lasers will not resume for 20-30 ms.

\section{BEAM DIAGNOSTICS}

The viewing (or collection) optics for this system is designed so that the volume observed is only slightly larger than the laser beam. At the narrowest point, the observed volurne is approximately $5.7 \mathrm{~mm}$ across. The measured divergence of the lasers is approximately $620 \mu \mathrm{rad}$, so with the $5000 \mathrm{~mm}$ focal length lens which is used to focus the beam into the scattering volume, the $1 / \mathrm{e}^{2}$ point of the beam waist is $3.1 \mathrm{~mm}$. Therefore, a tolerance of $\pm 1.3 \mathrm{~mm}$ in alignment (and divergence effects) must be maintained.

To help maintain proper alignment, a computer controlled feedback alignment system and divergence monitoring systems are used. The laser system is situated a distance from the DIII-D Tokamak. To deliver the beam through the machine, a beam path with total length of $35 \mathrm{~m}$ is needed. ${ }^{3} \mathrm{~A}$ helium-neon laser is adjusted so that it is colinear with the beam pack. This beam defines the beamline for purposes of alignment. Position sensitive detectors (PSD) are located behind the last two mirrors in the beamline. These two mirrors are located on either end of a 12 foot long granite 
bench, which is firmly attached to the structure which holds the collection lens. This provides a very stable structure to use as a reference for maintaining alignment with this system. The mirrors which direct the beamline toward either cletector are motor driven and controlled by a dedicated computer. The position of the beam after the system has been aligned is programed into the computer, and the computer adjusts each mirror to maintain this set position.

Since only long term drifts in the beamline are of interest for feedback alignment, a low pass filter is used to provide a slow response output from the PSD amplifier. To check the colinearity of the HeNe to the Nd:YAG beam pack, a fast output from the amplifiers is used. The pulses from the multiple firing of the lasers are integrated, and are then averaged to find the center of the pack. This is then compared to the position of the $\mathrm{HeNe}$ beari as seen by the slow output and compared to determine if any drift between the two beams have occurred. This information can be used to adjust the HeNe beam by moving it back into colinearity with the Nd:YAG beam pack with computer controlled mir. irs to correct for any drift which may occur.

Due to the asynchronous firing of the lasers, some variations in thermal lensing or stress induced birefringence of the rods could occur. These effects would result in a change in the beam divergence. To monitor this, a variation of the standard pinhole test will be used. A 1-D reticon with fast read out electronics, will be situated at the focus of a sampled portion of the laser beams. From this, the divergence can easily be obtained for every laser pulse. 
Misalignment of the collection lens with respect to the beam could occur from mechanical distortions and vibrations of the support structures from the magnetic: fields of the DIII-D tokamak. A system to monitor the alignment of the collection lens is under development. This system monitors the position of the image of the laser beam at the image plane of the collection lens. This lens has a magnification of $\sim 0.25$ at the lens center, so the beam image width is $\sim 1.5 \mathrm{~mm}$. A set of five 400 micrometer diameter fiber optics that form a row across the laser beam image (Fig. 5) is placed at the top, middle and bottom of the image. The lens is properly aligned when the image falls onto the middle three fibers, and misalignment can easily be recognized when the image encroaches onto either of the outside fibers. The fibers are connected to silicon avalanche photodiodes, whose signal is digitized by a gated integrator. Light which is scattered from Thomson scattering, or Rayleigh scattering during calibration, is seen by these fibers, and used to determine if any drift has occurred since the initial alignment.

\section{ACKNOWLEDGMENTS}

The authors gratefully acknowledge H. Rohr and H. Murmann of the Max-Plank Institut fúr Plasmaphysik for sharing their experience of $\mathrm{Nd}$ :YAG based Thomson scattering systems, and their ideas for future systems. This work was sponsored by the U.S. Department of Energy under Contract No. DE-AC03-89ER51114. 


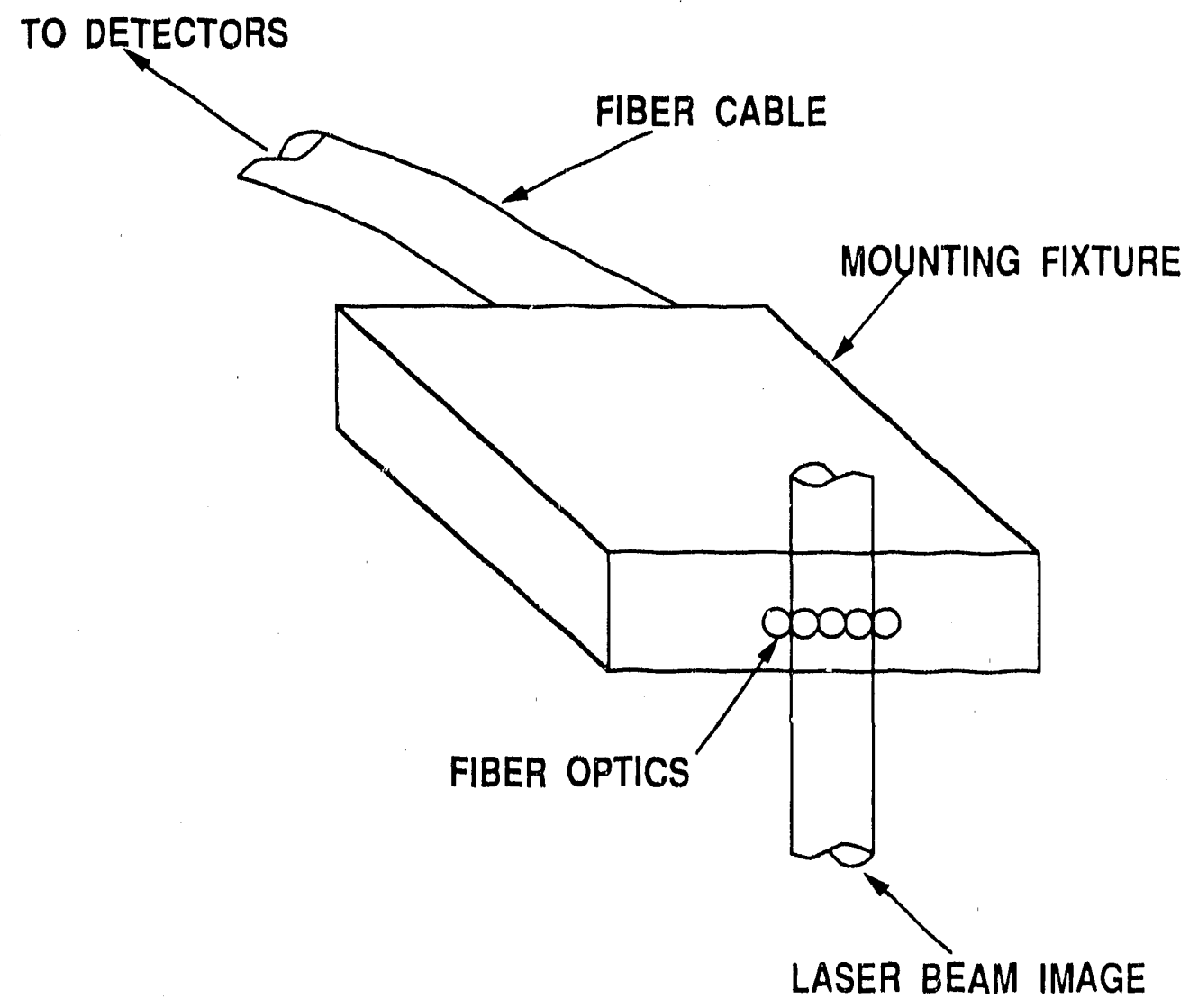

Fig. 5. Fiber optic arrangement for the detection of the image position of the scattered light forrned by the collection lens. The image falls on the center three fibers.

\section{REFERENCES}

1 E. Funfer, B. Kronast, H. J. Kunze, Phys. Letts. 5, 125 (1963).

2 H. Rohr, K. H. Steuer, D. Meisel, H. Salzmann, Nucl. Fusion 22, 1099-1102 (1982).

3 C. L. Hsieh, R. Chase, J. C. DeBoc, R. G. Evanko, P. Gohil, R. T. Snider, and R. E. Stockdale, Rev. Sci. Instrum. 591467 (1988). 

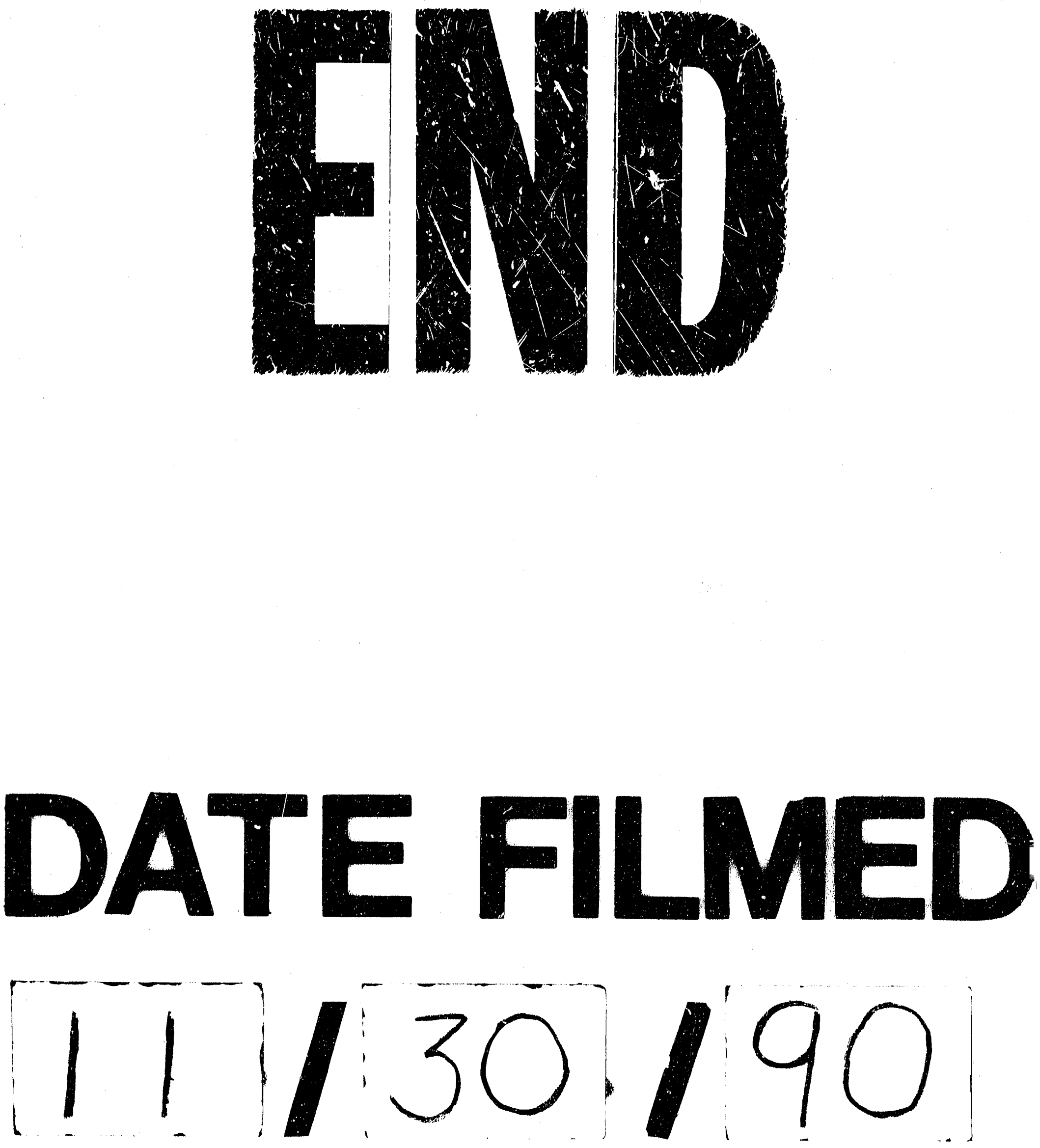
- 